\title{
«Von jagender Hast und vorzeitigem Zusammenbruch»1 Zur Psychodynamik schleichender Veränderungen in beschleunigten Zeiten
}

\author{
Benigna Gerisch (Berlin)
}

Zusammenfassung: In diesem Beitrag gehe ich zunächst der Frage nach, wie und auf welche Weise sich globale Beschleunigungsprozesse einschliesslich verknappter zeitlicher Ressourcen sowie Flexibilisierungs- und Mobilitätsanforderungen und der Gebrauch digitaler Technologien auf die Lebens- und Erfahrungswelt unserer Patienten und Patientinnen auswirken. Zugleich möchte ich zeigen, wie sich diese Dynamisierungsprozesse der letzten Jahre gleichsam wie unbemerkt in den Berufsalltag des Psychoanalytikers eingeschlichen haben. Und ferner nehme ich, vor dem Hintergrund der These eines konstitutiven Zusammenhangs von Beschleunigung und Perfektionierung, die Art und Weise in den Blick, wie sich infolge soziokulturell gewandelter Bedingungen spezifische Phänomene herauskristallisieren, wie zum Beispiel die narzisstische Akzentuierung des Körpers, die sich entlang eines Kontinuums von gesund hin zu pathologisch verorten lassen. Anhand einiger klinischer Fallvignetten werden diese theoretischen Überlegungen illustriert.

Schlüsselwörter: Selbstmanagementanforderungen, digitale Liebessemantiken, (autodestruktive) Körperpraktiken, Beschleunigung, Perfektionierung

\section{$1 \quad$ Einleitung}

Wenn wir uns zunächst vergegenwärtigen, dass die Beschleunigung in der Spätmoderne, wie sie in technischer Hinsicht insbesondere durch die rasante Steigerung von Mobilität und Kommunikationsgeschwindigkeit angestossen wurde, eine der folgenreichsten Wandlungen des 20. Jahrhunderts darstellt (Blumenberg, 1986; Elias, 1988; Geissler, 1999), dann, so der einhellige Befund, treffen wir auf sozialer, kultureller und psychischer Ebene auf ein signifikant verändertes Zeiterleben, einschliesslich der von Koselleck (2000) konstatierten «Beschleunigung des Erfahrungswandels» (vgl. King \& Gerisch, 2009). 
Zugleich wies der Kulturwissenschaftler Hartmut Böhme (2009) darauf hin, dass, historisch betrachtet, gesellschaftliche Umbrüche immer schon auch als Folge von Beschleunigungsdynamiken erfahren wurden, denen sich die Subjekte nach einer gewissen Zeit der Verstörung mehr oder minder gelungen angepasst hätten. Denn zumeist wurden entsprechende Wandlungen schon bald als verheissungsvolles Fortschrittsversprechen begrüsst. Der Eintritt der Kultur in die ultimative Geschwindigkeit der Spätmoderne zeichne sich nun jedoch in einschneidender Weise dadurch aus, dass gegenwärtig eine transhumaneWelt entstehe: Da beispielsweise die technische Architektur, die mathematische Modellierung und der interne Datenverkehr mit einer Geschwindigkeit arbeiteten, die jeglicher Anschauung entzogen sei, überschreite diese Technokultur die Dimension des Menschlichen. Eine ubiquitäre, tachogene Weltfremdheit werde erzeugt und begünstige zugleich Adaptionsstrategien in Gestalt von Unsterblichkeitsphantasmen, die-bezogen auf die Jenseitsreligionen - historisch vertraut und doch neu seien: Mehr denn je begäben sich die Menschen in die andere Wirklichkeit des Cyberspace und vertrauten auf die damit verbundenen Verheissungen, die suggerieren, dass die eigentliche Welt nicht die unsere sei, sondern erst jenseits der unseren beginne.

Mehr oder minder unstrittig ist also der Befund, dass wir es mit einer gleichwohl noch zu präzisierenden «neuen Dimension» (der Überschreitung des Menschlichen bezogen auf Technikentwicklungen, Mobilitäts- und Flexibilisierungsanforderungen usw.) in all ihren Abschattierungen zu tun haben, deren Auswirkungen ex negativo formuliert gleichsam als Angriffe auf menschliche Grundbedürfnisse wie Bindung, Verlässlichkeit, Sicherheit, Transparenz und Kontinuität interpretiert werden können, die kehrseitig aber ebenso eine weit gespannte und verführerische Projektionsfläche für (narzisstische) Omnipotenzfantasien bilden (vgl. Aubert, 2009).

Veränderungen, die uns künftig noch beträchtlicheVerstehensanstrengungen auch im psychoanalytischen Alltagsgeschäft abverlangen werden und implizit auf eine transdisziplinäre Perspektivierung verweisen: «Entscheidend scheint mir jedoch, dass - trotz aller Schwierigkeiten der Vermittlung - neuere sozial- und kulturwissenschaftliche Techniktheorien sich an dieser Stelle mit der psychoanalytischen Kulturtheorie treffen in der Annahme, dass Technikgebrauch ebenso wie Technikentwicklung nicht nur Bestandteil zweckrationaler und ökonomischer Zusammenhänge sind, sondern auch Ausdrucks- und Darstellungsmittel menschlicherWunsch- und Alpträume. Hier sehe ich eine wichtige Anschlussstelle für die psychoanalytische Forschung. Ein zweiter Berührungspunkt und eine zweite Anschlussstelle ergibt sich daraus, dass in den Sozial- und 
Kulturwissenschaften durchaus gesehen wird, dass die neuen Informations- und Kommunikationstechnologien nicht nur mit gesellschaftlichen, wirtschaftlichen, kulturellen Veränderungen einhergehen, sondern auch die Verfasstheit menschlicher Subjektivität tangieren» (Löchel, 2005, S. 170).

In meinem Beitrag möchte ich zunächst einerseits der Frage nachgehen, wie und auf welche Weise sich diese globalen Dynamisierungsprozesse einschliesslich verknappter zeitlicher Ressourcen sowie Flexibilisierungs- und Mobilitätsanforderungen in die psychoanalytische Alltagswelt gleichsam wie unbemerkt eingeschlichen haben. Und andererseits nehme ich, vor dem Hintergrund eines konstitutiven Zusammenhangs von Beschleunigung und Perfektionierung, die Art undWeise in den Blick, wie sich infolge soziokulturell gewandelter Bedingungen spezifische Phänomene herauskristallisieren, wie zum Beispiel die narzisstische Akzentuierung des Körpers.

Wenn ich aus eigenen Erfahrungen der letzten Jahre schöpfe, so bin auch ich zwar unentwegt mit der nachhaltigen Wucht von Beschleunigungs- und Mobilitätsdynamiken konfrontiert, die ich aber bemerkenswerterweise in ihren Auswirkungen nicht adäquat ins tägliche Tun «übersetzt» habe. Zwar nahm ich wahr, dass sich manche Termin- und Settingabsprachen schwieriger gestalteten als noch vor etwa zehn Jahren, aber über weite Strecken war ich stets geneigt, diese als Ausdruck der je spezifischen Psychopathologie des Patienten zu interpretieren. Konträr zur metatheoretischen Beschäftigung mit Beschleunigungs- und gesellschaftlichen Dynamisierungstheorien verlor ich in meinem konkreten Berufsalltag den Einfluss eben dieser äusseren Wandlungen aus dem Blick. So, als dürfte ich als Psychoanalytikerin der äusseren Realität nie dieses Gewicht verleihen wie unserem eigentlichen Arbeitsfeld: der intrapsychischen Seelenlandschaft unserer Patienten.

Gleichwohl prägen Flexibilisierungs-, Mobilitäts- und Perfektionierungsanforderungen, befristete Arbeits- und Mietverhältnisse - oder plötzliche Kündigungen - längst die Bildungs- und Erwerbsbiografien unserer Patienten und Patientinnen. Studierende müssen ihr Studium durch mindestens zwei Auslandssemester oder -praktika optimieren; Angestellte und Arbeiter können nicht mehr mit Langzeitverträgen rechnen, die Befristung und Kurzlebigkeit auch von Liebesverhältnissen ist längst zur Normalität geworden, und das Segment des höheren Managements steht gleichsam paradigmatisch für das, was wir hypermobiles Jet-Set-Leben nennen. Nichts ist und kann mehr verlässlich auf «Dauer gestellt werden» (vgl. King, 2009) - und die Zunahme der Kontingenzerfahrung - das heisst, die fundamentale Ungewissheit der eigenen Lebensperspektive - ist eine der signifikantesten und einschneidendsten Veränderungen der letzten Jahre. 
Im Kontext des Berufsalltages des Psychoanalytikers halten wir beharrlich dagegen mit unseren Behandlungsstandards der Kontinuität, hoher Frequenz, stabiler, verlässlicher, lang andauernder Arbeitsbeziehung und unserem Credo, dass psychische Entwicklungen und Psychoanalysen ihre Zeit brauchen. Angesichts der rasanten gesamtgesellschaftlichen Veränderungen mutet dieser psychoanalytische Wertekanon antiquiert und obsolet an, was kehrseitig keineswegs zur Folge haben sollte, diesen preiszugeben. Aber wir werden wohl oder übel gezwungen sein, uns mehr, als dies bisher auch innerhalb der Fachgesellschaften der Fall gewesen ist, mit diesen Veränderungen auseinanderzusetzen, sie als Teil einer sozialen Realität zu begreifen, die sich tief greifend in die individuellen Lebensentwürfe einschreibt und/oder diese mit zum Teil fatalen Folgen zur Erosion bringt. Und wir werden uns über Überbrückungsstrategien (Skype, Telefon, Mail usw.) angesichts ungleich häufigerer berufsbedingter Abwesenheitszeiten Gedanken machen müssen, bei allem Unbehagen, das auch mich angesichts dieser potentiellen Settingveränderungen ergreift. Aber dies kann uns letztlich nicht von unserem originären psychoanalytischen Tun abbringen: nämlich stets und konsequent nach der Bedeutung zu fragen, ob dies nun vorzeitige Beendigungen, Unterbrechungen oder Alternativsettings betrifft.

Löchel bemerkte bereits in einem Aufsatz von 2005: «Im eigenen Alltag hat indessen jeder von uns in den vergangenen Jahrzehnten tief greifende Umbrüche im Schreiben, Lesen, Recherchieren, Archivieren, Korrespondieren usw. erlebt und dabei eine Fülle von Selbstbeobachtungen machen können. Nur das analytische Nachdenken darüber ist bisher - von wenigen Ausnahmen abgesehen - informell und unsystematisch geblieben» (S. 169). In der Tat: Mehr unter vorgehaltener Hand, als handele es sich um einen skandalösen Tabubruch, erfährt man hier und dort, dass ein Kollege schon längst mit Patienten Skypesitzungen bei längeren Unterbrechungen durchführt, seine Terminansprachen inzwischen gern über SMS regelt und über dieses Medium mit seinen (gefährdeten) Patienten auch im Urlaub in Kontakt bleibt.

Ganz öffentlich hingegen haben erst kürzlich Kächele und Buchholz (im Druck) in ihrer Arbeit «Eine Notfall-SMS-Intervention bei chronischer Suizidalität - wie Konversationsanalyse klinische Beobachtung bereichert» infolge der längeren - vom Patienten und dem Psychoanalytiker - berufsbedingten Abwesenheit eindrucksvoll dargestellt, wie gerade die Knappheitsbedingungen der SMS-Konversation besondere affektive Verdichtungen gestatteten, wie ein Arbeitsbündnis aufgebaut werden könne und schliesslich, wie die Operationsweise der psychoanalytischen Deutung sichtbar werde und sogar Abstufungen von 
Empathie beschrieben werden könnten. Zugleich aber warnen die Autoren davor, die neuen elektronischen Medien zu einer «eigenen» Therapieform stilisieren zu wollen und raten, viele weitere Untersuchungen dieser Art abzuwarten (vgl. S. 1).

\section{Schleichende Veränderungen}

Paradigmatisch für die schleichenden Veränderungen sind die zunehmenden Komplikationen, zeitnahe Terminabsprachen zu organisieren, einen angemessenen psychoanalytischen Behandlungsrahmen und ein tragfähiges Arbeitsbündnis zu installieren, aber auch der exzessive Gebrauch von digitalen Kommunikationstechnologien insbesondere adoleszenter wie spätadoleszenter Patienten und Patientinnen, der in spezifischerWeise auch die Behandlungsstunden prägt und strukturiert.

Anfang letzten Jahres stellte sich eine 30-jährige selbstständige Fotografin in meiner Praxis wegen einer «Burnout-Symptomatik» und einer unheilvollen Dreiecksgeschichte vor, unter der sie sehr litte. Sie liebe diesen Mann über alles, aber er wolle sich für sie nicht von seiner Freundin trennen. Vor einigen Wochen habe sie einen ernsten Zusammenbruch erlebt: Sie habe nur noch im Bett gelegen, nicht mehr aufstehen können und stundenlang geheult. Eine Seite in ihr habe den Zusammenbruch kommen sehen, eine andere Seite habe diesen verleugnet, «solange ich noch arbeiten kann, wird's schon gehen», so ihr Fazit. Sie skizzierte dann eindrucksvoll die Plötzlichkeit, die Unmittelbarkeit des Zusammenbruchs, der sich unterschwellig schon lange angekündigt, sie dann aber doch «kalt erwischt» habe. Entweder, so ihr Entschluss, «ich bringe mich um oder ich tue etwas für mich», letzteres hätten ihr auch die Freunde geraten.

Sie liess sich dann aber doch eineWeileZeit, um Hilfe nachzusuchen, während sie wiederum in ihrer ersten Mail an mich die Dringlichkeit ihrer Hilfebedürftigkeit betonte, die, so das Signal, keinen Aufschub dulde. Aufgrund ihrer freiberuflichen Tätigkeit, der sie allmählich wieder nachging, und die ständig mit Auslandsreisen verbunden ist, stellte sich dieVereinbarung eines Erstgespräches als ausgesprochen schwierig dar. Schliesslich kam es erst vier Wochen nach ihrem Zusammenbruch zu einem ersten Termin. Zunächst schilderte sie anschaulich, was sie im Moment am meisten belaste, unterbrochen von heftigen Weinkrämpfen, und fügte dann wohl informiert - rasch an, dass sie unbedingt eine Psychoanalyse machen wolle, aber die nächsten Wochen nur sporadisch dafür Zeit habe. Sie sei in zwei grössere Projekte eingebunden, die sie auch schon aus finanziellen Gründen nicht «sausen lassen könne». Überdies sei man in ihrem Job schnell «weg vom Fenster», vor allem, wenn man grosse, einschlägige Kunden nicht adäquat bediene. Ich versuchte 
zunächst, ihre Ambivalenz im Hinblick auf eine längerfristige Behandlung in den Blick zu rücken. Deutungen, auf die sie nicht einging, sondern ausnahmslos mit dem Verweis auf ihre Selbstständigkeit parierte. In der Gegenübertragung nahm ich ihr prekäres Dilemma wahr: ihre fraglose Behandlungsbedürftigkeit einerseits und den äusseren Druck, auch zu funktionieren und für den Lebensunterhalt zu sorgen, andererseits. Ich empfand eine zunehmende Ausweglosigkeit, da ich keinen Weg sah, diese druckvolle Situation konstruktiv aufzulösen. Je mehr ich darauf insistierte, dass sich in dieser dilemmatösen Inszenierung eine intrapsychische Wiederholung verbergen könnte, umso verzweifelter wurde sie, da sie sich in ihrer aktuellen beruflichen Situation ganz und gar unverstanden fühlte. Schliesslich konnten wir uns darauf einigen, den Behandlungsbeginn um einen Monat zu verschieben einschliesslich ihres ernsthaft bekundeten Bemühens, für die Zeit der Psychoanalyse möglichst nur Aufträge am Heimatort anzunehmen. Interessanterweise war eine solche Lösung erst in dem Moment möglich geworden, als ich - auch im Sinne einer inneren Haltung - ihre prekäre berufliche Situation anzuerkennen vermochte.

In einem weiteren Fall spitzte sich die Situation einer Patientin kurz vor Weihnachten dergestalt zu, dass ihr und vielen anderen in einer grossen Dienstleistungsagentur «von heut auf morgen» gekündigt wurde. Für sie war diese Kündigung eine Katastrophe, da sie sich lange Zeit sehr schwer getan hatte, sich im Beruf wie auch in Liebesbeziehungen konstant und verlässlich auf etwas einzulassen. Stets hatte sie in einem borderlinetypischen Agieren «von heut auf morgen» alles hingeschmissen, wenn es zu ersten Konflikten kam. In dieser Firma aber habe sie erstmalig richtig Fuss fassen, habe gute und stabile soziale Kontakte aufbauen können und sich in ihrer Arbeit sehr geschätzt gefühlt. Für sie sei die Kündigung der untrügliche Beweis dafür, dass es überhaupt keinen Sinn habe, sich zu verändern, sich anzustrengen und überhaupt noch irgendetwas zu wollen. Warum habe man sie ständig gelobt, ihr «Honig um den Bart» geschmiert, wenn man von einer Sekunde auf die andere auf ihre Mitarbeit verzichten konnte. Die Kündigung war der unabwendbare Beleg für ihre absolute Wertlosigkeit, zumal, und das war das Entscheidendste, sie nichts getan habe, sie sich nichts zu Schulden habe kommen lassen, was die Entlassung rechtfertigen würde. Sie verstand überhaupt nichts mehr und fühlte sich «von Gott und derWelt», einschliesslich meiner Person, betrogen. Über weite Strecken versuchte ich immer wieder, die faktische, inhumane Ungerechtigkeit der plötzlichen Kündigung einerseits und die innere Verarbeitungsweise andererseits zunächst nur zu benennen und zu differenzieren. Diese Differenz, die ich einzutragen versuchte, hörte sie gleichwohl stets 
nur als Legitimation des Agierens der Chefs. Erst allmählich öffnete sie sich der Perspektive, dass ihr fraglos Unrecht geschehen sei, ihre Kündigung die Folge einer eklatanten Misswirtschaft im Kontext gravierender globaler Veränderungen gewesen ist, dass dies aber nichts aussage über ihren Wert und ihre Bedeutung, die sie als Person habe.

Schliesslich bot man ihr einen Job mit einem gänzlich anderen Tätigkeitsbereich in einer kleinen Firmen-Dependance in einer $500 \mathrm{~km}$ entfernten Stadt an. Da sie verschuldet war und sich keine "Auszeit» nehmen konnte, Angst vor der Arbeitslosigkeit und den frustrierenden Bewerbungsgesprächen hatte, die sie schon zahllos absolviert hatte, und ihr Freund erst vor kurzem ebenfalls dorthin versetzt worden war, entschied sie sich - bedauerlicherweise - dafür, die Behandlung zu beenden und wegzuziehen.

Fraglos kann man beide Verläufe präzise und allein entlang der Psychodynamik und intrapsychischen Welt der Patientinnen entfalten; natürlich kämpften sie Zeit ihres Lebens mit einer quälenden Selbstwertunsicherheit, tiefgreifenden Ambivalenzen und Spaltungen, einer eklatanten Frustrationsintoleranz und der Neigung zum Agieren.

Aber mir ging es hier darum zu zeigen, wie und auf welche Weise sich gravierende globale Veränderungen, die Firmen und Betriebe rasant in die Insolvenz treiben oder über interne Optimierungszwänge zu flächendeckenden Kündigungen führen, zur Folge haben, dass diese Umbrüche eben nur noch bedingt institutionell abgefedert werden und ganz wesentlich von den Individuen selbst integriert und ausbalanciert werden müssen. Mehr noch: Je nach intrapsychischer Verfasstheit werden diese Erfahrungen eruptiv als individuelles Scheitern und Versagen verbucht und produzieren Erosionen und Zusammenbrüche, die dringend einer Behandlung bedürfen, die aber wiederum mit Verweis auf die bedrohliche äussere Situation nicht in Anspruch genommen werden kann oder vorzeitig beendet werden muss.

Letzteres Beispiel verweist zudem eindrücklich auf das, was Hartmut Rosa (2005) als «Tendenz zu einer spätmodernen Form situativer Identität» (S. 373) ausgemacht hat und sich in seiner Formel, «Man ist heute nicht mehr Bäcker, sondern man arbeitet als Bäcker», prägnant zusammenfassen lässt. Das heisst, wenn das «soziale Rasen das biografische Rasten» nicht mehr zulässt, dann sind die nachmodernen Subjekte gezwungen, sich permanent neu zu erfinden, oder anders gesagt: Im Unterschied zur zeitresistenten Biografie ist gegenwärtig nur noch die Performanz des Subjekt- und Lebensentwurfes «auf Dauer gestellt». 
An diesen Befund anknüpfend hat Rosa eine Differenz von Surfern und Drifterni.S. unterschiedlicher Reaktions- undVerarbeitungstypologien ausgemacht. Während der Surfer, getragen von einem unerschöpflichen Omnipotenzgefühl, es als eine narzisstische Herausforderung erlebt, sich auf nichts mehr festzulegen, um sich immer wieder neu zu erfinden, der immer Ausschau nach der nächsten tollen Welle hält, sei es der verheissungsvolle Job in New York oder Shanghai, so wird der Drifter, zu dem meine Patientin zählen kann, von den Wellen hin- und her geschleudert, er wird grob gezwungen, sich fremdbestimmt zu verorten, z. B. wenn er einfach entlassen wird, bis hin, dass er schliesslich gänzlich den Boden zu verlieren droht.

\subsection{Vom Segen und Fluch der Smartphones}

Des weiteren ist zu beobachten, dass der exzessive Gebrauch moderner Kommunikationstechnologien (Handy, Smartphone) und die Installation eines «second life» in sozialen Netzwerken den Alltag, die Erlebensweisen, die Beziehungsgestaltungen und letztlich auch die Behandlungsstunden $u$.a. von Patienten und Patientinnen mit katastrophischen Trennungs- und Deprivationserfahrungen, panischen Verlassenheitsängsten und Ungetrenntheitssehnsüchten aufbemerkenswerteWeise präfiguriert. Die digitalen Kommunikationstechnologien liessen, so Bergmann (2005), nicht nur Entfernungen «im Raum und in der Zeit schrumpfen», sondern sie steigern zugleich «die kommunikative Erreichbarkeit in bislang ungeahntem Mass und machen Abwesende $\mathrm{zu}$ - virtuell - Anwesenden» (S. 4).

Diese Patienten vertrauen überdies nicht mehr ihrer eigenen Erinnerung, ihrer subjektiven Narration, sondern das Handy und die in ihm gespeicherten Daten werden gewissermassen als Beweismittel genutzt und gebraucht. Nur bleibt oft gänzlich unklar, um welche Beweisführung es sich eigentlich handelt.

Frau A., einst verlassen und gequält von Mutter, Vater und Stiefvater, wollte mir immer wieder ihre verworrene Liebesgeschichte zu S. anhand ihrer gespeicherten SMS-Dialoge begreifbar machen. Mehr noch: In mir sah sie nur mehr eine Interpretin gleich einer Handleserin oder Wahrsagerin der epischen Dialoge, die ich mit Sinn, Bedeutung und Erläuterung auffüllen sollte, einschliesslich des drängenden Wunsches, Prognosen über den Verlauf der Beziehung abzugeben ${ }^{2}$. In meinem Bemühen, auf die Erzählung zu rekurrieren, ihr Erleben zu fokussieren, reagierte sie verstört und manchmal mit fassungslosen Tränen. Und das, obwohl mir Frau A. selbst erklärte, dass nirgends so viel gelogen und betrogen werde wie mit diesen Kommunikationstechnologien: Da kann man posten, so empörte sie 
sich, gestern Nacht in der coolsten Disco der Stadt gewesen zu sein, obwohl man verheult und einsam auf dem Sofa sass.

Sie konnte nicht anders, als das Leben von S., das er nun ausserhalb von ihr führte, panisch und paranoisch zu verfolgen. Es fiel ihr ausgesprochen schwer, ihre obsessive Lebensüberwachung von S. während der Behandlungsstunden zu unterbrechen. Möglicherweise verpasste sie genau in diesen 50 Minuten eine existentiell wichtige Botschaft. Sie empfand meine Aufforderung, einfach nur zu erzählen, was in ihr vorginge, als grausam.

Sie stürzte in schwere Krisen, wenn sie sah, dass er sich bei Facebook herumtrieb, mit anderen kommunizierte, sich aber mit keiner Silbe an sie wandte. Einen schweren, suizidalen Zusammenbruch erlitt sie, als S. kurze Zeit später seinen Beziehungsstatus bei Facebook änderte und sich als "getrennt» positionierte, obwohl er ihr gegenüber so eindeutig von Trennung noch gar nicht gesprochen hätte. Zu ähnlichen Krisen kam es, wenn sie nachvollziehen konnte, dass er eine verzweifelte SMS von ihr zwar erhalten und auch gelesen hatte, eine ersehnte Antwort aber ausblieb. Anschaulich beschrieb sie ihre Abhängigkeit von den akustischen Signalen des Smartphones: Hörte sie anhand eines individuell eingerichteten Tons, dass eine Nachricht eingegangen war, stets verknüpft mit der sehnsuchtsvollen Hoffnung, sie möge von S. sein, dann war sie wieder in der Welt, erhielt sie das Recht auf Existenz, setzte sich ihr Leben wieder in Bewegung; blieb das ersehnte Signal aber aus, so versank sie allmählich in eine abgrundtiefe Leere, umfangen von einer grauen, todesähnlichen Starre. Je länger sie nichts hörte, umso auswegloser erlebte sie sich in diesem unbegrenzten Raum, in dem sie sich an nichts mehr zu halten und zu befestigen vermochte.

Eindrücklich hat bereits Bergmann (1982) aufgezeigt, dass unerwartete Schweigephasen, die in der Regel nicht paradigmatisch für die interaktive Organisation von SMS-Dialogen seien (vgl. Günthner, 2012), vom Sender nahezu immer als verstörend und beunruhigend erlebt würden: «Dieses «Nichts», das auf seine sequenzinitiierende Äusserung folgt, offenbart ja als solches nicht, was es bedeutet oder worin es seinen Grund hat. Das Ausbleiben einer konditionell relevanten Folgeäusserung zwingt daher den Sequenzinitiator dazu, sich auf die Suche nach dem Grund oder der Bedeutung dieses «Nichts` zu machen. Vom Erfolg bzw. Misserfolg dieser Suche wird es abhängen, ob und in welcher Weise der Sequenzinitiator von sich aus mit einer pausenterminierenden Äusserung eingreift» (Bergmann, 1982, S. 156).

Übersetzten wir diesen nüchternen Befund des Konversationsanalytikers Bergmann in die psychodynamische Konzeption der inneren Welt unserer 
Patienten, so erhellt sich uns augenblicklich das Phänomen, dass eben gerade jene Patienten und Patientinnen wie Frau A. eine spezifische Affinität zur Technik der Unmittelbarkeit haben und zugleich in eklatanter Weise anfällig und überaus verzweifelt auf das als Vernichtung erlebte Unterlaufen der Jetzt-und-SofortKommunikation reagieren.

Zugleich war verblüffend, wie sie selbst die Kunst der Verzögerung - die «Redezugvakanz», so Bergmann (1982) - beherrschte: Minutiös schilderte sie mir, wie lange und unter welchen Bedingungen sie mit einer Antwortet wartete. Das Hightech-Gerät der Kommunikations-Unmittelbarkeit wurde gleichsam mittels der Antwortpause und Verzögerung strategisch im verworrenen Liebesspiel eingesetzt, um sich, so ihre Begründung, einen letzten Rest von Würde, Souveränität und Autonomie zu bewahren.

Sie klärte mich begeistert über die Vielzahl technischer Raffinessen auf, die ihr Smartphone bereithielt, das auf eine mir gänzlich unbekannte und offenbar neue Weise Beziehungen, Sehnsüchte, Nähe und Distanz, Wünsche und Ängste zu regulieren vermochte. So könne sie sich selbst einen Erinnerungscode installieren, der sicherstellte, die Nummer von S. auf keinen Fall zu wählen; das sei in "Ausnahmesituationen» wichtig, wenn man zum Beispiel sturzbetrunken in einem Club sei. Nichts peinlicher, so ihr Kommentar, als wenn man aus Versehen die Nummer des Angebeteten wählte, der von einem im Moment nichts mehr wolle. Oder es sei möglich, über eine bestimmte Funktion den Kontakt zum Anderen zu blocken, worüber der wiederum eine Nachricht erhalte. Eine neue Beziehungsregulationssprache taucht auf: Da ist von Blocken, Löschen, Stummstellen, Statuseinstellungen, Online-Offline-Signalen usw. die Rede. Und jede dieser Funktionen wiederum hat fraglos eine Bedeutung, die etwas über den aktuellen Stand der Beziehung, etwas über das Beziehungsgefüge aussagt. Aber angesichts der Vielzahl an Regulationsmechanismen ist eben die Frage nach der tieferen, unbewussten Bedeutung kaum mehr möglich.

Da diese Patienten ohnehin zeitlebens darauf angewiesen waren, intensivst die Welt ihrer Objekte zu «scannen», um potentielle Gefahren und Bedrohungen zu antizipieren, so wird auch das Handy im Sinne eines Teilobjektes hinsichtlich seiner impliziten und verrätselten Botschaften unentwegt untersucht und «gedeutet». Erschwerend kommt in diesem Prozess hinzu, dass die Fülle an Funktionen suggeriert, dass es keine Eindeutigkeit (mehr) gebe und man im günstigen Falle noch hoffen dürfe. In der Uneindeutigkeit liegt gleichermassen etwas Verwirrendes wie Tröstliches: «Wenn er wirklich nichts mehr von mir wollte, wenn er wirklich nicht will, dass ich mich melde, dann hätte er mich längst geblockt», erläuterte 
mir Frau A. Ob die Frage nach Zusammensein oder Getrenntsein noch Bedeutung hat oder definitiv keine mehr wird nun anhand der technischen Funktionen überprüft und ausgelotet. Da bleibt kaum Raum, sich über die erlebte Qualität einer Beziehung - und deren Ende - Gedanken zu machen, Fantasien zu entwickeln, die sich unabhängig vom «technischen Beweismittel» einstellen könnten. Der konkretistische Gebrauch des Gerätes mit seiner Fülle an Einstellungsfunktionen korrespondiert fraglos mit einem konkretistischen Erleben der Beziehungsgestaltung, der Wünsche, Ängste und intrapsychischen Katastrophenszenarien. Vor allem die Sehnsüchte nach Ungetrenntheit und Dauerpräsenz werden einerseits maximal erfüllt und zugleich, in Trennungs- und Verabschiedungssituationen, andererseits brutal konterkariert. Es ist gewissermassen die unentwegte, masochistisch grundierte Vergegenwärtigung, dass der andere lebt und wieder liebt, ich, die Andere, aber «entsorgt» wurde, nicht mehr teilhabe, nur noch mittelbar, als Cyberspace-Verfolgerin.

Dies liesse sich verstehen im Sinne eines reaktualisierten Erlebens des deprivierten Säuglings, dessen Mutter irgendwie und irgendwo in dieser Welt ist, aber nicht verfügbar für die Bedürfnisse, Nöte und Katastrophen des Kindes. Schon immer, so formulierte es Frau A., habe ich mich später gefragt: Wo war sie eigentlich? Mit wem, wie konnte sie so leben, während sie mich zu Hause, damit ich nicht weglaufen konnte, ans Bett gebunden hatte?

So bleibt sie virtuell stets mit dem Objekt verklebt, es entsteht kein Raum zur Trennung, zur Trauer, zum Loslassen, zum Nachdenken über die Wiederholung des inneren Schreckens, sondern vielmehr verharrt sie in einer pausenlosen Überprüfung der Bewegungen des Anderen, die wiederum phantasmatisch und destruktiv gegen das eigene Selbst gewendet und aufgeladen werden: Warum war er solange bei Facebook, mit wem hat er gesprochen, wer hat ihm geschrieben usw.? Warum wendet er sich mit keiner Zeile mehr an mich?

Nirgends eindringlicher als in diesen Behandlungen wurden mir die Raffinesse und das Risiko dieser digitalen Technologien bewusst, oder, wie es Frau A. schlagfertig formulierte: Ist eine Beziehung gut und stabil, sind Handys ein Segen, geht etwas schief oder zerbricht sie, dann sind sie ein Fluch.

Eine der grossen Herausforderungen in solchen Behandlungen besteht darin, sich passager auf diesen Konkretismus einzulassen, zugleich aber sich stetig darum zu bemühen, die Fülle an multideterminierten Bedeutungsebenen zu dechiffrieren. Vielleicht verbirgt sich - jenseits der psychopathologischen Konnotation - in den technischen Codes und deren subjektiver Verwendung ja eine neue, geheime Semantik der Liebe, die wir selbst noch längst nicht verstanden 
haben, die wir aber vielleicht in Metaphern übersetzen, sie auf diese Weise wieder verflüssigen könnten. «Manchmal können wir», so Buchholz (2013), «eben mithilfe von Metaphern, dann doch das sagen, was wir nicht denken können - und nutzen dabei das, was ich einmal als die «transzendente Funktion` der Metapher beschrieben hatte: eine gute Metapher nutzt die Sprache und weist dabei beständig über die Sprache hinaus» (S. 6; Hervorhebung im Original).

\section{$3 \quad$ Zum konstitutiven Zusammenhang von Beschleunigung und Perfektionierung}

Während gegenwärtig zahlreiche soziologisch-sozialpsychologische Diagnosen eine Veränderung der Temporalstrukturen in Richtung zunehmender Beschleunigung (z. B. Rosa, 2005) verdeutlicht haben, finden sich zugleich Hinweise auf eine wachsende Tendenz zu Effektivierung und Optimierung, die insgesamt als gesellschaftliche Diskurse der Perfektionierung (z. B. Sandel, 2007) bezeichnet werden können. Als ein wesentliches Ergebnis kann die Feststellung eines konstitutiven Zusammenhangs von Beschleunigung und Perfektionierung gelten (vgl. King \& Gerisch, 2009). Ein Perfektionierungsstreben, das in signifikanterWeise die Indienstnahme des Körpers und somit eine neue Dimension der Hochkonjunktur des Körpers produziert.

Einschränkend möchte ich bereits an dieser Stelle hervorheben, dass ich nicht davon ausgehe, dass die äussere Welt ursächlich die Symptomatologien, Syndrome und Phänomene der inneren Welt produziert, wohl aber eine imposante Matrix und Schablonen für Prädispositionen liefert, in denen sich die intrapsychischen Konfliktfelder primär und körpernah unbewusst verhaken. Das heisst, dass die regressive Tendenz zur Instrumentalisierung des Körpers, zum Körperkonkretismus und der konkretistische Gebrauch der Kommunikationstechnologien im Zuge der aktuellen gesellschaftlichen Veränderungen von der Industrie- zur Mediengesellschaft mit ihrer Kultur der Vergleichzeitigung auf eklatante Weise pseudoprogressiv begünstigt und potenziert wird.

Vereinfacht formuliert wird der Jetzt-und-sofort-Imperativ des Säuglings, der zwischen Augenblick und Dauer noch nicht differenzieren kann, in extenso durch die Kultur der Unmittelbarkeit bedient. Die Paradoxien stellen sich ferner folgendermassen dar: Durch die aktuellen strukturellen Veränderungen einschliesslich ihrer Technologien werden genau die Anforderungen und Voraussetzungen unterlaufen - wie die Anerkennung von Differenz, Getrenntheit, Begrenztheit, von Vergänglichkeit und Endlichkeit -, die für psychisches Wachstum und emotionale Reife unabdingbar sind. Das heisst konkret: Die Anerkennung 
von Differenz wird durch die Verflüssigung von Beziehungen und die rasante Objektaustauschbarkeit unterlaufen, die von Getrenntheit durch die allzeit verfügbaren Kommunikationstechnologien, die von Begrenztheit durch omnipotente Möglichkeitsräume und multifunktionale Identitätsoptionen und die der Vergänglichkeit und Endlichkeit durch Umbaumassnahmen am menschlichen Körper mittels Body- und Neuroenhancement.

Mehr noch: Spätmoderne Entwicklungen, insbesondere die Kommunikationstechnologien und bodyshaping-Varianten bieten sich als universelle Verführungsinstrumente an und suggerieren, sie würden die psychischen Bedingungen für emotionales Wachstum gleichsam kompensieren und psychisches Arbeiten obsolet werden lassen. Und doch artikuliert sich unter der Hand beziehungsweise unter der Haut ein Unbehagen, das sich in seinerVerschränkung von intrapsychischen und gesellschaftlichen Dynamiken selbst nicht mehr versteht, aber auf die Leerstellen und Defizite zurückverweist, die mittels moderner Technologien aufzufüllen versucht werden und schliesslich im Sinne einer Figur von Mehr-Desselben einen sich pausenlos verfehlenden, exzessiv-süchtigen Konsum eben dieser Technologien erzeugen (vgl. Gerisch, 2009).

Wenn man also von der gegenwärtig kontrovers diskutierten Frage absieht, ob es tatsächlich eine Veränderung der primären seelischen Struktur gegeben hat (vgl. Reiche, 1991; Dornes, 2010), die wir vorerst nur als hypothetische Prognose beantworten können, so können wir durchaus einen Wandel der phänomenologischen und thematischen Ausgestaltung uns vertrauter und bekannter Symptomatologien und Pathologien der Moderne konstatieren. Der Mensch leidet gegenwärtig weniger unter einer lustfeindlichen Sexualmoral, die bei Freuds Hysterikerinnen noch jene bizarren proteusartigen Verwandlungskünste des Körpers hervorbrachten, sondern unter diffusen Identitätszerklüftungen. Oder, wie es Ehrenberg (1998) konstatiert, am erschöpfenden Imperativ, er selbst zu sein. Der zur Flexibilität und Beschleunigung genötigte Mensch der Spätmoderne leidet vor allem unter verhüllten oder konkreten Zukunftsängsten, Orientierungslosigkeit, Bindungslosigkeit und Fragmentierung - im Kontrast zu den scheinbar unbegrenzten Möglichkeitsräumen. Das viel beschworene Ideal von Machbarkeit und Freiheit des modernen Kapitalismus wurde zum Fluch von individueller Selbstoptimierung; und das globale Gefühl der Entwurzelung und Entbindung produziert kehrseitig die imposante Wiederkehr gleichermassen des makellosen wie des zerstörten Körpers. Ein Körper, der untrennbar mit den Erfahrungen von Abhängigkeit, Bedürftigkeit und Unverfügbarkeit verknüpft bleibt. Im Taumeln zwischen Überflutung und Deprivation, zwischen Beschleunigung und Entgrenzung, 
zwischen Ohnmachtserleben und Omnipotenzimperativ, zwischen Bindung und Entbindung, zwischen Zeit- und Raumnot, wird der Körper gleichsam als drittes, identitätsbefestigendes Objekt zu optimieren versucht, wie zugerichtet.

Ich schliesse mich in diesem Zusammenhang der These von Küchenhoff (2005) an, der konstatiert, dass der Körper der Gegenwart mit der zentralen Aufgabe der Identitätsbildung befrachtet und damit überfordert werde, indem "Anforderungen an eine gelingende Lebenspraxis, an Sinnstiftung und Selbstverwirklichung [...] auf den Umgang mit dem eigenen Körper verschoben» (S. 175) werden. Küchenhoff präzisiert diesen Befund, indem er die Hochkonjunktur des Körpers in den Kontext des Verlustes bzw. derVerkümmerung interpersonell-intersubjektiver Erfahrungsdimensionen stellt: «Dadurch wird die narzisstische Dimension des Körpererlebens immer wichtiger, auf Kosten der anderen Dimensionen». Das imaginäre Körperbild droht andere Formen des Körpererlebens zu überlagern. Mit anderen Worten: «Die Beanspruchung des Körpers für die Stabilisierung von Identitätsgefühlen führt komplementär zu einem Verkümmern der anderen Erfahrungsdimensionen» (ebd.), die Küchenhoff unter der Begriffstrias «Unverfügbarkeit», «körperliche Intersubjektivität» und «Kreativität» differenziert ausfaltet.

Dieses Ausmass an narzisstischen Akzentuierungen scheint nicht folgenlos zu bleiben: Denn während wir einerseits über ungeahnte technisierte Körperperfektionierungsmassnahmen verfügen, die das regressive Phantasma der Realitätsverleugnung bedienen und einen omnipotenten Körperkonkretismus erzeugen, müssen wir auf der anderen Seite die Zunahme gravierender, sprachloser Selbstzerstörungspassionen verzeichnen.

Auf der Seite des sich zunächst nicht als Krankheit oder Symptom artikulierenden Körperkultes - im Zuge einer sich stetig perfektionierenden Enhancementpolitik - finden wir die boomende Schönheitschirurgie (vgl. auch Rohde-Dachser, 2007) und dekorative long-life Kosmetik, Körperschmuck wie Tätowierungen und Piercings, Diäten- und Schlankheitswahn, Anti-AgingProgramme bis hin zur medizinisch-neurologischen Manipulation in Form so genannter Hirnschrittmacher. Überdies ist ein enormer Anstieg des Konsums von synthetischen Drogen insbesondere bei Jugendlichen und Lifestyle-Drogen bei Erwachsenen zu verzeichnen.

Demgegenüber steht die rasante Zunahme von Körperpathologien, d.h. intrapsychischen und soziokulturell überformten Konflikten, die im, am und mit dem Körper ausagiert werden. Hier treffen wir auf riskantes und selbst- oder fremdschädigendes Körperhandeln mit seinen geschlechtsspezifischen Ausgestaltungen: 
Bei jungen Frauen, mit grosser Deutlichkeit ab der Adoleszenz, überwiegen internalisierende Störungen wie Essstörungen, selbstverletzendes Verhalten und Suizidversuche, diffuse psychovegetative Störungen sowie Depressionen, aber auch Schönheitsoperationen (vgl. Gerisch, 2003, 2006; King, 2002, 2003).

Es sind überdies überdurchschnittlich häufig Frauen, die ihren als unzureichend und mangelhaft erlebten Körper als ursächlich für Kränkungen, Zurückweisungen und Objektverluste halten. Junge Männer hingegen tendieren eher zu Externalisierungen, etwa im Sinne von gewalttätigem oder dissozialem Verhalten, zu riskantem Handeln in Alltag, Sport und Strassenverkehr sowie zum Drogenkonsum.

Insbesondere der spätmoderne Körperkult erzeugt auf paradoxe Weise gleichsam eine «Dialektik der Selbstvervollkommnung»: In dem Masse, wie Selbstperfektionierung untergründig auf diskursiv beförderten Varianten der Verleugnung oder Nicht-Anerkennung der «facts of life» (Money-Kyrle, 1971) basiert, kann das Streben nach Selbstperfektion in Selbstzerstörung umschlagen (vgl. auch Ach \& Pollmann, 2006; Gerisch 2003, 2006).

An anderer Stelle haben King und ich (2008) bereits ausgeführt, dass sich das Unbehagen im Körper der (späten) Moderne mit seinen imposanten Beschleunigungs- und Entgrenzungsdynamiken präzise folgendermassen zusammenfassen lässt:

Wir treffen auf eine nahezu allseits präsente «Verführung zum Agieren», zum technologisch ermöglichten Unterlaufen von Integrations-, Reifungs- und Trauerprozessen etwa in Form von körpermodifzierenden, manipulativen Anti-Aging-Programmen und Schönheitsoperationen, die zudem sozial positiv sanktioniert wird. Diese Umarbeitungsstrategien mit ihren impliziten Verheissungen von ewiger Jugend und Unsterblichkeit liegen gleichsam quer zu den lebenslangen Anforderungen an psychische Integrationsprozesse, die auf der Anerkennung von Begrenzung und Endlichkeit basieren - und eben produktiv nur aus dieser Anerkennung zugleich ihre Fähigkeit zu symbolischer Überschreitung, zu Sublimierung und kreativer Veränderung gewinnen können. (Gerisch \& King 2008, S. 269) 


\subsection{Geschundene Seelen in perfekten Körpern}

Inzwischen sind wir an das Bild der Manager und (Fern)-Pendler in ICEs, in Bahnhöfen, Flughäfen und Flugzeugen, ausgestattet mit ihren Prothesen der Macht - Laptop, Tablet und Smartphone - längst gewöhnt. Fit, ewig jung und dynamisch jagen sie durch die Welt, scheinbar unangekränkelt an Leib und Seele, sie überwinden offenbar mühelos timeslots und zeigen beim nächsten Meeting keine Spur eines Jetlags. Zugleich wissen wir, dass die Gesundheitsvor- und Selbstfürsorge nirgends so eingeschränkt und suboptimal ist wie bei Pendlern und hypermobil Arbeitenden; und dass die gesundheitlichen, das heisst sowohl die psychischen als auch die somatischen Risiken im Vergleich zur nicht pendelnden Normalbevölkerung beträchtlich sind (vgl. Häfner, Rapp \& Kächele, 2012). Häfner und Mitarbeitende (2012) kamen in einer empirischen Untersuchung zu dem Ergebnis, dass der hohen Prävalenz psychischer Störungen bei Fernpendlern ein wenig ausgeprägtes bzw. inadäquates Inanspruchnahmeverhalten von psychosozialen Versorgungsangeboten gegenüberstehe, sodass bei beruflichem Pendeln von einer erheblichen Unter- bzw. Fehlversorgung ausgegangen werden müsse (vgl. S. 345).

Auch die soziale Integration erodiert bei den meisten, die ständig und für lange Zeit unterwegs sein müssen, um ihr Arbeitspensum zu schaffen. Zugleich aber tunen sie ihre Körper, um sich für ihre nächsten erschöpfenden Einsätze funktionstüchtig erleben zu können. Aber auch dieses «Leben auf der Überholspur» mit der Ausbeutung der gestählten und perfektionierten Körper produziert irgendwann seine Grenzen.

So kommen immer häufiger Patienten in unsere Notfallsprechstunden, die unter Ausschöpfung all dessen, was die Lifestyle-Medizin zur Selbstoptimierung so zu bieten hat, ihren Körper und seine Leistungsfähigkeit bis zum unvermeidbaren Kollaps auszureizen versucht haben und nun aber, unter dem Druck der kaum noch zu bewältigenden Anforderungen, von uns erneut die schnelle Lösung erwarten: «Bis Montag spätestens muss ich wieder fit sein, da muss ich in die USA zu einem Meeting», so die imperative Forderung eines fünfundfünfzigjährigen Managers. Jeder Versuch, diesen Teufelskreis von Karrieredruck und Kollaps durch ein psychotherapeutisches Angebot, das Zeit und Raum für einen Denk- und Verstehensprozess eröffnen könnte, zu durchbrechen, wird barsch als esoterisches Psychotherapeutengefasel vom Tisch gefegt: «Ich will nicht verstehen, warum mein Körper streikt, er soll wieder funktionieren.» Dieser Patient berichtete mir, dass er seine Hotels weltweit inzwischen nicht mehr nach dem kulinarischen, sondern nach den Fitnessangeboten aussuche. Er würde vor und nach den Meetings, egal, 
wo in der Welt, viel Zeit damit verbringen, seinen Körper fit zu halten. Ansonsten, so seine Befürchtung, würde er sein Pensum gar nicht mehr schaffen. Manchmal vergesse er darüber sogar seinen obligatorischen Anruf zu Hause, um sich nach dem Befinden seiner Frau und den Kindern zu erkundigen.

In dem Masse, wie die Gefahr zu scheitern ebenso gross ist, wie die soziale Norm, es nicht zu dürfen, wird der Körper in seiner Dysfunktionalität, vermeintlichen Hässlichkeit, Bedürftigkeit und Untauglichkeit auf der Matrix einer intrapsychischen Potenzierung als verfolgendes Objekt erlebt und verantwortlich gemacht für das potentielle oder reale Versagen und dafür mit noch radikaleren Disziplinierungsanstrengungen bestraft.

Es wird in der Regel ein rasch wirkendes Psychopharmakon gefordert oder bestenfalls ein sozial längst positiv konnotiertes Coaching erwartet, nicht aber ein langwierigerVerstehensprozess, der mit entwertenden Gesten abgetan wird. Auch darauf hat der Markt mit seiner Fülle an pseudopsychotherapeutischen CoachingAngeboten seit langem reagiert. ${ }^{3}$ Inzwischen geben Besserverdienende jährlich Unsummen für so genannte Personal Trainer aus, die im privaten Setting aus den «geschmähten Leibern geschönte Körper» (Ettl, 2006) zu modellieren versuchen.

Hier scheint ein zentraler Aspekt wiederzukehren, der von Sarasin (2001) entlang des Hygienediskurses des 18. und 19. Jahrhunderts bereits differenziert ausgearbeitet wurde. Gemäss der Devise: «Sorge Dich um Dich selbst: Wer den Reiz kontrolliert, beherrscht seinen Körper», konstituierte sich paradigmatisch ein Selbstverantwortungs-Imperativ, der zugleich eine omnipotente Herrschaft über die Zeit eben dieses reizbaren, bedürftigen Körpers implizierte.

\subsection{Vom Scheitern am Perfektionierungsideal}

Auch bei unseren Patientinnen und Patienten, die ernsthaft um Hilfe nachsuchen, treffen wir zunehmend auf eine beunruhigende narzisstische Akzentuierung des Körpererlebens und auf ein ausgeprägtes Selbstoptimierungsund Perfektionsstreben, das durch gegenwärtige Optimierungsdiskurse und Körperperfektionierungsimperative begünstigt und aufrechterhalten wird. Damit eng verknüpft ist der persistierende Widerstand, die Anstrengungen psychischen Arbeitens einschliesslich zu leistender Integrations- und Anerkennungsanforderungen auf sich zu nehmen.

In den letzten Jahren zeichnete sich in den Klagen insbesondere suizidaler Patientinnen und Patienten die narzisstische Akzentuierung des Selbstund Körpererlebens deutlich ab, wenn vom Scheitern, Verfehlen, Versagen, Untauglichkeit, chronischen Selbstzweifeln usw. die Rede ist, im Unterschied zur 
bislang hohen Relevanz des Objektverlustes in der suizidalen Psychodynamik. So etwa Frau B. im Erstgespräch: «Jetzt habe ich mein gesamtes Leben umgekrempelt, habe gesund gegessen, keinen Alkohol mehr getrunken, viel Sport gemacht, und nun habe ich schon wieder einen Infekt. Ich will diesen Körper einfach weghaben!» Oder Herr K.: «Ich packe das Studium einfach nicht mehr, komme nicht mehr mit, obwohl ich nicht blöd bin. Nun jogge ich exzessiv, zu irgendwas muss ich mich ja zwingen. Hilft aber auch nicht, ich will nur noch tot sein.»

Von besonderer Bedeutung ist die zunehmende Radikalisierung der Selbstoptimierung und des rasanten Umschlags in Selbstzerstörung. Eine Dynamik, die uns von Popstars wie Michael Jackson und Amy Winehouse, wie aber auch von narzisstischen und Borderline-Persönlichkeitsstörungen hinlänglich vertraut ist. Und die bei Suizidalen, insbesondere bei Adoleszenten und Spätadoleszenten, eine beunruhigende Ausgestaltung annimmt. Mehr noch: Gerade bei Suizidalen treffen wir häufig auf ein ausgeprägtes, narzisstisch grundiertes Selbstoptimierungsund Perfektionsstreben, eine grosse Intoleranz gegenüber Asymmetrien und Dysbalancen und, wie es bereits Henseler (1974) beschrieb, nur scheinbar paradox, auf hypochondrisch ausgestaltete Todesängste. Was sich im Zuge der von mir behaupteten Dialektik von Selbstvervollkommnung und Selbstzerstörung im Kontext der Suizidalität als neue Dimension abzeichnet, ist einerseits die eklatante Verführung durch Körperperfektionierungstechniken der spätmodernen Gesellschaften, auf deren impliziten Optimierungsdiskurs Suizidale in besonderer Weise anzusprechen scheinen, und andererseits der damit eng verknüpfte persistierendeWiderstand, die Anstrengungen psychischen Arbeitens einschliesslich zu leistender Integrations- und Anerkennungsanforderungen in Kauf zu nehmen. Das heisst, die Fetischisierung einzelner Körperteile, sowie kehrseitig deren vernichtende Verurteilung, greift unbewusst in die fragile Übergangsphase einer sukzessiven Aneignung einer Körperrepräsentanz ein, wenn wir davon ausgehen, dass der Säugling sich nicht von Anfang an als körperliche Einheit erlebt, sondern er seinen Körper ursprünglich als in all seinen Gliedern fragmentiert und als ausserhalb seiner Selbst existierend wahrnimmt. Der Körper (bzw. Körperteile) ist somit das erste Objekt, das zunächst vor allem als Quelle von Dysphorie und Unwohlsein wahrgenommen wird, wenn nämlich die innere Homöostase durch Hunger, Kälte oder andere Missempfindungen gestört ist. Eine erste psychische Reifeaufgabe besteht darin, den Körper allmählich als zu sich selbst gehörig zu erleben und die Partialobjekte als Ganzes zu integrieren. Dieser Prozess ist nicht nur immer schon intersubjektiv präfiguriert, sondern er erweist sich auch als ein lebenslang überaus störanfälliges Projekt. 
Ausgehend von dieser Matrix ist eine meiner leitenden Annahmen, dass der spätmoderne Körperkult nicht nur eine Auftrennung von Leib und Körper begünstigt, wie es Ettl (2006) formulierte, sondern durch die Hypertrophierung, Fetischisierung und Idealisierung einzelner Körperteile, die es zu perfektionieren oder gar gänzlich zu tilgen gilt, intrapsychischen Spaltungsprozessen, die paradigmatisch für die suizidale Psychodynamik sind, Vorschub leistet bzw. diese aufrechterhält. Es ist fraglos evident, in welchem Ausmass die Kultivierung von narzisstischen Oberflächenartikulationen (vgl. auch Melzer, 1974), einschliesslich der Verleugnung von Zeitgebundenheit, durch den modernen Körperkult produziert wird. In der Weise, dass alle ästhetisierenden wie destruktiven Körperpraktiken auf einer intensiven sensorischen, zunächst überwiegend schmerzhaften Körpererfahrung basieren. Und zweitens: In beiden Varianten sind Manipulationen und Angriffe auf das Zeitgefüge, zum Beispiel hier: der Vergänglichkeit und dort: der psycho-physiologischen Reifung zu verzeichnen. Schliesslich drittens: Das überbordende Bombardement medial konstruierter und vermittelter, virtuell bearbeiteter, perfekter Körper kolonisiert auch die tiefen Schichten des Unbewussten und bietet kollektiv Identifikationsvarianten mit Imitationskörpern an, die nicht mehr sind als transhumane, entleerte Bildschablonen.

Ich möchte dafür ein kurzes Beispiel geben: Seit Jahren behandle ich wöchentlich in einer vierstündigen Analyse eine hoch begabte, attraktive, chronisch suizidale, japanische Spitzensportlerin, inzwischen 25-jährig. Abgesehen von ihren extremen Leistungs- und Perfektionsansprüchen, ist sie primär von ihrem Körper, aktuell von ihren vermeintlich abstehenden Ohren gequält, die eben diesen Anforderungen nicht genügen. Da sie es in der Pubertät durch selbstmanipulative Praktiken bereits geschafft habe, ihren Körper in ein anorektisches Korsett zu zwingen, wolle sie nun unbedingt durch konstanten, symmetrischen Druck auf ihre Ohren die ersehnte Symmetrie erzielen. Diese manipulativen Modifikationsversuche nehmen zwanghafte Züge insbesondere in Ruhezuständen wie beim Lesen oder beim Fernsehschauen an. Da es ihr aber partout nicht gelinge, das gewünschte Resultat zu erzwingen, surfe sie manisch getrieben durchs Internet, auf der Suche nach kostengünstigen, operativen Eingriffen. Meine kontinuierlichen Versuche, die psychische Bedeutung dieses Erlebens zu untersuchen, drohten immer wieder zu scheitern. «Da ich mich eines Tages sowieso umbringe, möchte ich dann wenigstens so schön sein, wie ich es mir immer wieder gewünscht habe».

Zusammengefasst ist angesichts der skizzierten Befunde von zentraler Bedeutung, dass gegenwärtig die «Lösung» immer häufiger in der technisch machbaren Körperperfektionierung gesucht wird und nicht etwa, wie bereits ausgeführt, 
in dem mühevollen Weg der psychischen Arbeit im Sinne der Dechiffrierung der komplexen Dimensionen unbewussten, intrapsychischen Erlebens und seiner Bedeutungen einschliesslich der damit untrennbar verknüpften Bedeutung des Anderen.

\section{Literatur}

Ach, J. S. \& Pollmann, A. (Hrsg.) (2006). Einleitung. In Dies. No body is perfectBaumassnahmen am menschlichen Körper. Bioethische und ästhetische Aufrisse (S. 9-17). Bielefeld: transcript.

Aubert, N. (2009). Dringlichkeit und Selbstverlust in der Hypermoderne. In V. King \& B. Gerisch (Hrsg.), Zeitgewinn und Selbstverlust. Folgen und Grenzen der Beschleunigung (S. 87-100). Frankfurt a. M.: Campus.

Bergmann, J. (1982). Schweigephasen im Gespräch. Aspekte ihrer interaktiven Organisation. In H.G. Soeffner (Hrsg.), Beiträge zu einer empirischen Sprachsoziologie (S. 143-184). Tübingen: Narr.

Bergmann, J. (2005). Editorial. In Psychotherapie und Sozialwissenschaft. Zeitschrift für qualitative Forschung und klinische Praxis. Themenheft: «Beratung und Therapie per Internet und Handy», 7(2), 3-8.

Blumenberg, H. (1986). Lebenszeit und Weltzeit. Frankfurt a. M.: Suhrkamp.

Böhme, H. (2009). Full Speed - Slow Down: Ambivalenzen der Moderne. In V. King \& B. Gerisch (Hrsg.), Zeitgewinn und Selbstverlust. Folgen und Grenzen der Beschleunigung (S. 63-84). Frankfurt a. M.: Campus.

Buchholz, M.B. (2013). Kulturträger - Archäologie, Architektur, Metapher. PsychoNews-Letter der DGPT, Nr. 94.

Dornes, M. (2010). Die Modernisierung der Seele. Psyche - Zeitschrift für Psychoanalyse und ihre Anwendungen, 64, 995-1033.

Elias, N. (1988). Über die Zeit. Arbeiten zur Wissenssoziologie II. Frankfurt a. M.: Suhrkamp.

Ettl, Th. (2006). Geschönte Körper - geschmähte Leiber. Psychoanalyse des Schönheitskultes. Tübingen: editon diskord.

Ehrenberg, A. (1992). Das erschöpfte Selbst. Depression und Gesellschaft in der Gegenwart. Frankfurt a. M.: Campus.

Gerisch, B. (2003). Die suizidale Frau. Psychoanalytische Hypothesen zur Genese. Göttingen: Vandenhoeck \& Ruprecht.

Gerisch, B. (2006). «Keramos Anthropos»: Psychoanalytische Betrachtungen zur Genese des Körperselbstbildes und dessen Störungen. In J.S. Ach \& A. Pollmann (Hrsg.), No body is perfect - Baumassnahmen am menschli- 
chen Körper. Bioethische und ästhetische Aufrisse (S. 131-161). Bielefeld: transcript.

Gerisch, B. (2009). Körper-Zeiten: Zur Hochkonjunktur des Körpers als Folge der Beschleunigung. In V. King \& B. Gerisch (Hrsg.), Zeitgewinn und Selbstverlust - Folgen und Grenzen der Beschleunigung (S. 123-143). Frankfurt a. M., New York: Campus.

Gerisch, B \& King, V. (2008). Das Unbehagen im Körper der Moderne Transdisziplinäre Überlegungen zu geschlechtertypischen Körperpraktiken und Symptombildungen. In G. Schlesinger-Kipp \& R. P. Warsitz (Hrsg.), Die neuen Leiden der Seele - Das (Un-)Behagen in der Kultur (S. 260-271). Bad Homburg: Selbstverlag.

Geissler, K.H.A. (1999). Vom Tempo der Welt-und wie man es überlebt. Freiburg i. Br.: Herder.

Günthner, S. (2012). «Lumpf meinen Slumpf»: Die interaktive Organisation von SMS-Dialogen. In Ayass, R. \& Meyer, Ch. (Hrsg.), Sozialität in Slow Motion. Theoretische und empirische Perspektiven; Festschrift für Jörg Bergmann (S. 353-373).Wiesbaden: Springer VS.

Häfner, S., Rapp, H. \& Kächele, H. (2012). Psychosoziale Belastungen von Bahnpendlern - was soll man tun? Psychotherapeut, 57, 343-351.

Henseler, H. (1974). Narzisstische Krisen. Zur Psychodynamik des Selbstmords. Opladen: Westdeutscher Verlag.

Kächele, H. \& Buchholz, M. B. (im Druck). Eine Notfall-SMS-Intervention bei chronischer Suizidalität - wie Konversationsanalyse klinische Beobachtung bereichert.

King, V. (2002). Körperbedeutungen. In Dies. Die Entstehung des Neuen in der Adoleszenz. Individuation, Generativität und Geschlecht in modernisierten Gesellschaften (S. 159-200). Wiesbaden: Leske \& Budrich.

King, V. (2003). Der Körper als Austragungsort adoleszenter Konflikte. AKJP, Zeitschrift für Theorie und Praxis der Kinder- und Jugendlichen-Psychoanalyse und der tiefenpsychologisch fundierten Psychotherapie, 119(7), 321-342.

King, V. \& Gerisch, B. (2009). Einleitung. In Dies. Zeitgewinn und Selbstverlust Folgen und Grenzen der Beschleunigung (S. 7-17). Frankfurt a. M., New York: Campus.

Koselleck, R. (2000). Zeitschichten. Studien zur Historik. Frankfurt a. M.: Suhrkamp. Küchenhoff, J. (2005). Öffentlichkeit und Körpererfahrung. In Ders. Die Achtung vor dem Anderen. Psychoanalyse und Kulturwissenschaften im Dialog (S. 169-184). Weilerswist: Velbrück Wissenschaft. 
Löchel, E. (2005). Entgrenzung von Zeit und Raum im Kontext der neuen Medien (Einführung). In K. Münch, E. Löchel, I. Bataller et al. (Hrsg.), Zeit und Raum im psychoanalytischen Denken (S. 169-172). Bad Homburg: Selbstverlag. Melzer, D. (1974). Adhesive identification. In A. Hahn (Hrsg.) (1994), Sincerity and other works. Collected papers of Donald Melzer (S. 335-350). London: Karnac. Money-Kyrle, R. (1971). The aim of Psychoanalysis. International Journal of Psychoanalysis, 49, 103-106.

Rosa, H. (2005). Beschleunigung. Die Veränderung der Zeitstrukturen in der Moderne. Frankfurt a. M.: Suhrkamp.

Reiche, R. (1991). Haben frühe Störungen zugenommen? Psyche, 12, 1045-1066.

Rohde-Dachser, Ch. (2007). Zur Psychodynamik schönheitschirurgischer Körperinszenierungen. Psyche - Zeitschrift für Psychoanalyse und ihre Anwendungen, 61, 97-124.

Sarasin, Ph. (2001). Reizbare Maschinen. Eine Geschichte des Körpers 1765-1914. Frankfurt a. M.: Suhrkamp.

Sandel, M. J. (2007). The Case against Perfection. Ethics in the age of genetic engineering. Cambridge, London, Massachusetts: Harvard University Press.

\section{Anmerkungen}

1 Paraphrasiertes Zitat, zitiert nach Hartmut Böhme 2009, S. 77.

2 Ich fühlte mich an den Beitrag - Prenez soin de vous - des französischen Pavillons auf der Biennale in Venedig aus dem Jahre 2007 erinnert: Hier hat die Künstlerin, Sophie Calle, im Original die Abschiedsmail ihres letzten Freundes aufgehängt und dazu, entlang derWände des quadratischen Raumes, die zahllosen Kommentare und Interpretationen von über 100 Frauen verschiedenster Professionen, denen sie auf einer zweijährigen Reise quer durch das Land die Mail vorgelegt hatte, da ihr selbst, so ihre Begründung, keine Antwort eingefallen sei.

3 Dass Psychotherapien und Psychoanalysen insbesondere von strukturell schwerer gestörten Patienten, bei denen häufig nur der Körper «spricht», Zeit, unter Umständen viel Zeit brauchen, ist ein Argument, das im effizienzorientierten Beschleunigungskarussell als antiquiert abgetan wird und kaum noch Gehör findet. Denn auch die Kostenträger von psychotherapeutischen Behandlungen springen nur allzu gern auf das Phantasma zu beschleunigender Heilungsstrategien auf, indem wir nicht nur gehäuft mit Leistungskürzungen und -beschränkungen konfrontiert sind, sondern künftig mit der Nivellierung individuell indizierter unterschiedlicher Behandlungsmethoden einschließlich der spezifischen Behandlungsdauern zu rechnen haben. 